\title{
Thermodynamics of Field Theories from Spinning Branes *
}

\author{
T. Harmark and N.A. Obers ${ }^{\dagger}$ \\ Niels Bohr Institute and Nordita, Blegdamsvej 17, DK-2100 Copenhagen, Denmark \\ E-mail: harmark@nbi.dki, iobers@nordita.dk
}

ABStRACT: We discuss general spinning $p$-branes of string and M-theory and use their thermodynamics along with the correspondence between near-horizon brane solutions and field theories with 16 supercharges to describe the thermodynamic behavior of these theories in the presence of voltages under the R-symmetry. The thermodynamics is used to provide two pieces of evidence in favor of a smooth interpolation function between the free energy at weak and strong coupling of the field theory. (i) A computation of the boundaries of stability shows that for the D2, D3, D4, M2 and M5-branes the critical values of $\Omega / T$ in the two limits are remarkably close and (ii) The tree-level $R^{4}$ corrections to the spinning D3-brane generate a decrease in the free energy at strong coupling towards the weak coupling result. We also comment on the generalization to spinning brane bound states and their thermodynamics, which are relevant for non-commutative field theories.

\section{KEYwords: 'Duality in Gauge Field Theories, Black Holes in String Theory, p-branes, D-branes}

\section{Introduction}

The discovery that four-dimensional black holes have thermodynamic properties due to Hawking radiation [i] has been one of the main motivations to study the thermodynamics of black $p$-branes in string and M-theory [2]. Through the recently conjectured correspondence between the near-horizon limit of these brane solutions and quantum field theories in the large $N$ limit $\left[\begin{array}{l}3 \\ 3\end{array}, \overline{4}\right]$, it has become clear that this study not only probes the nature of quantum gravity, but also provides information about the thermodynamics of quantum field theories in the large $N$ limit [5in. In particular, for the non-dilatonic branes (D3,M2,M5) the near-horizon limit of the supergravity solutions has been conjectured [3in to be dual to a certain limit of the corresponding conformal field theories. For the more general dilatonic branes of type II string theory preserving 16 supersymmetries, similar duality relations have

\footnotetext{
* Talk presented by N.O. at Quantum aspects of gauge theories, supersymmetry and unification, Paris, France (Sept. 1-7, 1999).

†Work supported in part by TMR network ERBFMRXCT96-0045.
}

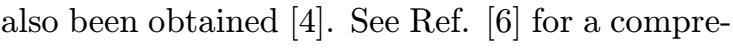
hensive review on the AdS/CFT correspondence

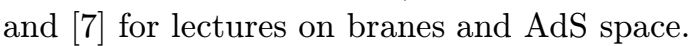

A common feature of these dualities between near-horizon backgrounds and field theories, is that the supergravity black $p$-brane solution exhibits an $S O(d)$ isometry (where $d=D-p-1$ is the dimension of the transverse space) which manifests itself as the R-symmetry of the dual field theory. As a consequence, by considering spinning black $p$-brane solutions, we expect on the one hand to learn more about the field theory side, and on the other hand, be able to perform further non-trivial tests of the duality conjectures that include the dependence on this R-symmetry group. The thermodynamics on the two dual sides, including a stability analysis, turns out to provide a useful starting point for this comparison. For the non-dilatonic branes this study has been initiated in a number of recent papers $\left[\overline{8}, \overline{9}, \overline{9}, \overline{1} 0_{1}^{\prime}\right]$ and was further generalized in $\overline{1} \overline{1} \overline{1}$ for all branes that are half-BPS solutions of string and M-theory. This work was extended in [12]1, which includes the first construction of spinning brane bound states along with their thermody- 
namics, which are relevant for non-commutative field theories. In this talk, we will summarize the

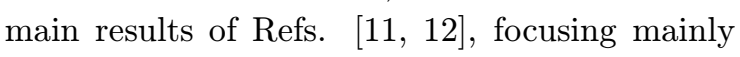
on the spinning $p$-branes, their thermodynamics and application to the supergravity/field theory correspondence, while commenting at the end on the spinning brane bound states.

\section{Spinning $p$-branes and the near- horizon limit}

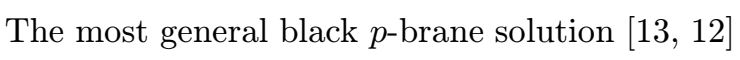
can be derived by oxidization from the rotating black hole solutions in [14]. We focus on general spinning black $p$-branes that are half-BPS solutions of string and M-theory (in the extremal and non-rotating limit), which include the M2 and M5-branes of M-theory and the D and NS-branes of string theory. The asymptotically-flat solution of a spinning black $p$-brane in $D$ dimensions takes the form

$$
\begin{aligned}
d s^{2}= & H^{-\frac{d-2}{D-2}}\left(-f d t^{2}+\sum_{i=1}^{p}\left(d y^{i}\right)^{2}\right) \\
& +H^{\frac{p+1}{D-2}}\left(\bar{f}^{-1} K_{d} d r^{2}+\Lambda_{\alpha \beta} d \eta^{\alpha} d \eta^{\beta}\right) \\
& +H^{-\frac{d-2}{D-2}} \frac{1}{K_{d} L_{d}} \frac{r_{0}^{d-2}}{r^{d-2}}\left(\sum_{i, j=1}^{n} l_{i} l_{j} \mu_{i}^{2} \mu_{j}^{2} d \phi_{i} d \phi_{j}\right. \\
& \left.-2 \cosh \alpha \sum_{i=1}^{n} l_{i} \mu_{i}^{2} d t d \phi_{i}\right)
\end{aligned}
$$

where the harmonic function $H$ is given by

$$
H=1+\frac{1}{K_{d} L_{d}} \frac{r_{0}^{d-2} \sinh ^{2} \alpha}{r^{d-2}}
$$

and we refer to [1] $\left.{ }_{1}^{1}\right]$ for further details, including the corresponding expressions of the dilaton $\phi$ and gauge potential $A_{p+1}$. Since the transverse space is $d=D-p-1$ dimensional, these spinning solutions are characterized by a set of angular momenta $l_{i}, i=1 \ldots n$ where $n=\operatorname{rank}(S O(d))$, along with the non-extremality parameter $r_{0}$ and another parameter $\alpha$ related to the charge.

Using standard methods of black hole thermodynamics we can compute the relevant thermodynamic quantities of the general solution. The mass $M$, charge $Q$ and angular momenta $J_{i}$ are determined by the background in the asymptotic region. The Hawking-Bekenstein entropy $S$ as well as all the intensive quantities, temperature $T$, chemical potential $\mu$ and angular velocities $\Omega_{i}$ are computed from the properties at the horizon. The final results are summarized by

$$
M=\rho_{d} r_{0}^{d-2}\left(d-1+(d-2) \sinh ^{2} \alpha\right)(2.3)
$$

$T=\frac{d-2-2 \kappa}{4 \pi r_{H} \cosh \alpha}, \quad S=4 \pi \rho_{d} r_{0}^{d-2} r_{H} \cosh \alpha$

$\mu=\tanh \alpha, \quad Q=\rho_{d} r_{0}^{d-2}(d-2) \sinh \alpha \cosh \alpha$

$\Omega_{i}=\frac{l_{i}}{\left(l_{i}^{2}+r_{H}^{2}\right) \cosh \alpha}, \quad J_{i}=2 \rho_{d} r_{0}^{d-2} l_{i} \cosh \alpha$

where $r_{H}$ is the horizon radius determined by

$$
\prod_{i=1}^{n}\left(1+\frac{l_{i}^{2}}{r_{H}^{2}}\right) r_{H}^{d-2}=r_{0}^{d-2}
$$

and $\kappa=\sum_{i=1}^{n} l_{i}^{2} /\left(l_{i}^{2}+r_{H}^{2}\right)$. We have also defined $\rho_{d}=V_{p} V\left(S^{d-1}\right) / 16 \pi G$ with $V_{p}$ the worldvolume of the $p$-brane and $V\left(S^{d-1}\right)$ the volume of the (unit radius) transverse $(d-1)$-sphere. The quantities (2.23) obey the conventional Smarr formula

$$
M=\frac{d-1}{d-2}(T S+\Omega \cdot J)+\mu Q
$$

which follows from the first law of thermodynamics and the scaling behavior of the extensive quantities.

Our main interest, however, is in the nearhorizon limit of these spinning branes, defined as follows: One introduces a dimensionfull parameter $\ell$ and performs the rescaling

$$
\begin{aligned}
& r=\frac{r_{\mathrm{old}}}{\ell^{2}}, \quad r_{0}=\frac{\left(r_{0}\right)_{\mathrm{old}}}{\ell^{2}}, \quad l_{i}=\frac{\left(l_{i}\right)_{\mathrm{old}}}{\ell^{2}} \\
& h^{d-2}=\frac{h_{\mathrm{old}}^{d-2}}{\ell^{2 d-8}}, \quad G=\frac{G_{\mathrm{old}}}{\ell^{2(d-2)}}
\end{aligned}
$$

where the new quantities on the left hand side are expressed in terms of the old quantities labelled with a subscript "old", and $h$ is defined by $h^{d-2}=r_{0}^{d-2} \cosh \alpha \sinh \alpha$. The near-horizon limit is then defined as the limit $\ell \rightarrow 0$ keeping all the new quantities in (2.6i) fixed. The rescaling $(2.6)$ is accompanied by appropriate rescalings of the fields $g, \phi, A$ such that the new ones are finite, which turns out to precisely leave the action invariant. The resulting near-horizon spinning $p$ - 
brane is [i] $\left.{ }^{1} \overline{1}_{1}^{\prime}\right]$

$$
\begin{aligned}
d s^{2}= & H^{-\frac{d-2}{D-2}}\left(-f d t^{2}+\sum_{i=1}^{p}\left(d y^{i}\right)^{2}\right) \\
& +H^{\frac{p+1}{D-2}}\left(\bar{f}^{-1} K_{d} d r^{2}+\Lambda_{\alpha \beta} d \eta^{\alpha} d \eta^{\beta}\right) \\
& -2 H^{-\frac{d-2}{D-2}} \frac{1}{K_{d} L_{d}} \frac{h^{\frac{d-2}{2}} r_{0}^{\frac{d-2}{2}}}{r^{d-2}} \sum_{i=1}^{n} l_{i} \mu_{i}^{2} d t d \phi_{i}
\end{aligned}
$$

where the harmonic function is now given by

$$
H=\frac{1}{K_{d} L_{d}} \frac{h^{d-2}}{r^{d-2}}
$$

and we refer to [1] 1in for the corresponding expressions of the dilaton and gauge potential.

The thermodynamics in the near-horizon limit is then

$$
\begin{gathered}
E=\rho_{d} \frac{d}{2} r_{0}^{d-2} \\
T=\frac{d-2-2 \kappa}{4 \pi r_{H}} \frac{r_{0}^{\frac{d-2}{2}}}{h^{\frac{d-2}{2}}}, \quad S=4 \pi \rho_{d} r_{0}^{\frac{d-2}{2}} h^{\frac{d-2}{2}} r_{H} \\
\Omega_{i}=\frac{l_{i}}{\left(l_{i}^{2}+r_{H}^{2}\right)} \frac{r_{0}^{\frac{d-2}{2}}}{h^{\frac{d-2}{2}}}, \quad J_{i}=2 \rho_{d} r_{0}^{\frac{d-2}{2}} h^{\frac{d-2}{2}} l_{i}
\end{gathered}
$$

where the internal energy is obtained from the energy above extremality $E=M-Q$. Note that since the charge and chemical potential have become constant these are not thermodynamic parameters anymore, so that the thermodynamic quantities are given in terms of the $n+1$ supergravity parameters $\left(r_{0}, l_{i}\right)$. Due to the different scaling behavior the near-horizon quantities (2.9) do not satisfy the Smarr law $(2.5)$, but instead the near-horizon Smarr law [1] 1]

$$
E=\frac{d}{2(d-2)}(T S+\Omega \cdot J)
$$

It is not difficult to obtain the energy function of the microcanonical ensemble in terms of the extensive variables using the horizon equation (2.4) and $(\underline{2} . \overline{9})$, yielding

$$
\begin{aligned}
E^{d / 2}= & \left(\frac{d}{2}\right)^{d / 2} \rho_{d}^{-(d-4) / 2} h^{-(d-2)^{2} / 2}\left(\frac{S}{4 \pi}\right)^{d-2} \\
& \cdot \prod_{i}\left(1+\left(\frac{2 \pi J_{i}}{S}\right)^{2}\right)
\end{aligned}
$$

Moreover, for any near-horizon spinning $p$-brane solution with $d$ transverse dimensions, the Gibbs free energy takes the simple form

$$
F=E-T S-\Omega \cdot J=-\rho_{d} \frac{d-4}{2} r_{0}^{d-2}
$$

Except for the case of one non-zero angular momentum, it is not possible in general to obtain a closed-form expression of $F$ in terms of the proper intensive quantities, the temperature $T$ and the angular velocities $\Omega_{i}$. However, in a low angular momentum expansion this change of variables can be achieved for the general spinning case to any desired order in $\omega_{i}=\Omega_{i} / T$, and will be sufficient for some of our applications below. We also remark that the Gibbs free energy ( $(2 . \overline{1} \overline{2})$ is properly reproduced [1] $\left.{ }_{1}^{1}\right]$ for all near-horizon spinning branes by computing the on-shell Euclidean action, taking into account the boundary term. This is not only an important consistency check but also essential when calculating string corrections to the free energy of spinning branes.

\section{Thermodynamics of dual field the- ories}

The thermodynamics of spinning branes and their near-horizon limit is interesting in its own right, but also enables us to obtain information on the thermodynamics of field theories in the strongly coupled large $N$ limit, via the near-horizon supe-

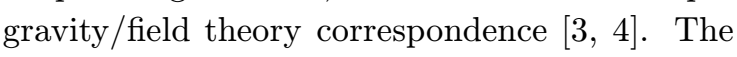
fact that the branes are spinning, introduces the new thermodynamic parameters $\Omega_{i}$ and $J_{i}$ on the supergravity side which are conjectured to correspond in the field theory to the voltage and charge under the $S O(d)$ R-symmetry group respectively. We can trust the supergravity description of the dual field theory, when the string coupling $g_{s} \ll 1$ and the curvatures of the geometry are small. This implies in all cases that the number of coincident $p$-branes $N \gg 1$. For the M2- and M5-brane this is the only requirement. while for the $\mathrm{D} p$-branes one must further demand that [i]

$$
1 \ll g_{\mathrm{eff}}^{2} \ll N^{\frac{4}{7-p}}, \quad g_{\mathrm{eff}}^{2}=g_{\mathrm{YM}}^{2} N r^{p-3}
$$

where for the purpose of the thermodynamics, one needs to set $r=r_{H}$ in the effective YM coupling $g_{\text {eff }}$. 
In view of this correspondence, we can write the Gibbs free energy and other thermodynamic quantities in terms of field theory variables. In particular we need to specify the relation between the parameter $\ell$ entering the near-horizon limit and the relevant length scale of the theory and compute the rescaled quantities in $\left(2 . \overline{6_{1}^{3}}\right)$. For the M2 and M5-branes the relation to the 11dimensional Planck length is $\ell=l_{p}^{3 / 4}$ and $l_{p}^{3 / 2}$ respectively, from which one obtains $h^{6} \sim N$ and $h^{3} \sim N$. For D-branes on the other hand, $\ell=l_{s}$ and one finds $G \sim g_{\mathrm{YM}}^{4}$ and $h^{d-2} \sim \lambda$, where $g_{\mathrm{YM}}$ is the Yang-Mills coupling constant and $\lambda=g_{\mathrm{YM}}^{2} N$ the 't Hooft coupling. Restricting to $\mathrm{D} p$-branes, the resulting expression for the (low angular momentum expansion of the) free energy $(\overline{2} . \overline{1} \overline{2})$ in field theory variables is then,

$$
\begin{aligned}
& F_{\mathrm{D} p}=-c_{p} V_{p} N^{2} \lambda^{-\frac{p-3}{p-5}} T^{\frac{2(7-p)}{5-p}}\left[1+\frac{S_{p}^{1}}{\pi^{2}} \sum_{i} \omega_{i}^{2}\right. \\
& \left.+\frac{S_{p}^{2}}{\pi^{4}}\left(\sum_{i} \omega_{i}^{2}\right)^{2}+\frac{S_{p}^{3}}{\pi^{4}} \sum_{i} \omega_{i}^{4}+\ldots\right]
\end{aligned}
$$

where we have defined $\omega_{i}=\Omega_{i} / T$ and the numerical coefficients $c_{p}, S_{p}^{a}$ can be found in Ref. [1 $1 \overline{1}_{1}$. Similar expressions for the M2 and M5 brane, proportional to $N^{3 / 2}$ and $N^{3}$ respectively, are given in this reference as well. The same mapping can be done for all other thermodynamic quantities (2.9) and we note that for the special value of $\kappa=\frac{1}{2}(d-2)$ the temperature vanishes, implying that besides the usual extremal limit describing zero temperature field theory, there also exists a limit in which the temperature is zero, accompanied by non-zero R-charges.

\section{Stability analysis}

We can now analyze the critical behavior

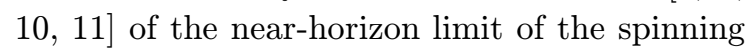
$p$-branes, which thus corresponds to the critical behavior of the dual field theories with non-zero voltages under the R-symmetry. The boundaries of stability have been computed in [i 11 in for both the GCE (grand canonical ensemble with thermodynamic variables $\left(T, \Omega_{i}\right)$ ) and the $\mathrm{CE}$ (canonical ensemble, with thermodynamic variables $\left.\left(T, J_{i}\right)\right)$ for $m \leq n$ equal non-zero angular momenta. To this end, we find the points in phase space at which the functions

$$
\begin{aligned}
& \mathrm{GCE}: \operatorname{det} \operatorname{Hes}(-F)=\frac{D_{S J}}{D_{T \Omega}} \\
& \mathrm{CE}: \quad C_{J}=T\left(\frac{\partial S}{\partial T}\right)_{\left\{J_{i}\right\}}=T \frac{D_{S J}}{D_{T J}}
\end{aligned}
$$

are zero or infinite, for the GCE and CE respectively. Here, we have denoted by $D_{T \Omega}$ the determinant $\frac{\partial\left(T, \Omega_{1}, \Omega_{2}, \ldots, \Omega_{n}\right)}{\partial\left(r_{H}, l_{1}, l_{2}, \ldots, l_{n}\right)}$ and likewise for $D_{T J}$, $D_{S \Omega}$ and $D_{S J}$. These determinants can be written as finite polynomials in $l_{i} / r_{H}=J_{i} / S$ and their zeroes will determine the boundaries of stability as $n$-dimensional submanifolds in the $(n+$ 1)-dimensional phase diagram. Hence they crucially depend on the fact that while there is a one-to-one correspondence between the $n+1$ supergravity variables and the extensive quantities $\left(S, J_{i}\right)$, the map to the intensive ones $\left(T, \Omega_{i}\right)$ or the mixed combination $\left(T, J_{i}\right)$ involves a noninvertible function.

In this talk, we restrict for simplicity to the case of one non-zero angular momentum in the GCE, in which case we find from ( $\bar{A} . \overline{1})$ that the region of stability (for $d \geq 5$ ) is determined by the condition

$$
J \leq \sqrt{\frac{d-2}{d-4}} \frac{S}{2 \pi}
$$

so that there is an upper bound on the amount of angular momentum the brane can carry in order to be stable. Put another way, at a critical value of the angular momentum density (which equals the R-charge density in the dual field theory) a phase transition occurs. The supergravity description also determines an upper bound on the angular velocity,

$$
\Omega \leq \frac{2 \pi}{\sqrt{(d-2)(d-4)}} T
$$

which is saturated at the critical value of the angular momentum. In Ref. [10 have been proposed for the nature of the resulting phase transition: D-brane fragmentation, in which the branes fly apart in the transverse dimension, and phase mixing in which angular momentum localizes on the brane. The latter is possible because from (2.9) it follows that for each value of $\Omega / T$ between 0 and the maximum in $(4.4)$, two pairs of supergravity variables $\left(r_{H}, l\right)$ 
can be found, one corresponding to a stable state and the other to an unstable state.

More generally, we remark that for general $d$, the two ensembles are not equivalent and that increasing the number of equal-valued angular momenta enlarges the stable region ${ }^{\ddagger 1}$. We also note that the case $d=4$ is special since the free energy (i2. $\overline{1} \overline{2}$ ) vanishes, and it is found that the temperature and angular velocity are not independent, so that the phase diagram is degenerate; for example for one non-zero angular momentum one finds the quadratic constraint

$$
(2 \pi T)^{2}+\Omega^{2}=h^{-2}
$$

Finally, we mention that the critical exponents for all spinning branes can be uniformly computed in both ensembles to be $1 / 2$, a value which satisfies scaling laws in statistical physics.

\section{Comparison to weak coupling}

An important question is to what extent do we observe the above stability phenomena in the large $N$ limit of the dual field theory, also at weak coupling. Extending the method of Ref. [i], one can obtain in an ideal gas approximation the free energies of the field theories for the case of the M-branes and the D-branes of type II string theory,

$F=-\tilde{c}_{p} V_{p} T^{p+1} \sum_{\vec{\alpha}} \operatorname{Li}_{p+1}\left[s_{\vec{\alpha}} \exp \left(-\sum_{i=1}^{n} \alpha \cdot \omega\right)\right]$

where the $S O(d)$ R-charge weights $\vec{\alpha}$ run over the 16 different particles and $s_{\vec{\alpha}}$ is +1 for bosons and -1 for fermions. The polylogarithms $\mathrm{Li}_{p+1}$ are not defined for real numbers greater than one, but can be continued to this region, so that the free energies can be expressed as exact power se-

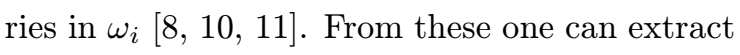
the stability behavior by considering again the zeroes of $\operatorname{det}(\operatorname{Hes}(F))$. The resulting critical values of $\omega$ are summarized in Table 5 , along with the corresponding values $(4.4)$ at strong coupling.

For zero angular momentum, it has been conjectured [1] 15 - $]$ that the free energy smoothly interpolates between the weak and strong coupling

\footnotetext{
${ }^{\ddagger 1}$ Except for the cases $d=8,9$, which have no stability boundary for one non-zero angular momentum.
}

\begin{tabular}{|c||c|c|}
\hline Brane & $\omega_{\text {weak }}$ & $\omega_{\text {strong }}$ \\
\hline D1 & Stable & 1.2825 \\
\hline D2 & 1.5404 & 1.6223 \\
\hline D3 & 2.4713 & 2.2214 \\
\hline D4 & 3.3131 & 3.6276 \\
\hline D5 & 4.1458 & Not defined \\
\hline D6 & 4.9948 & Unstable \\
\hline M2 & 1.5404 & 1.2825 \\
\hline M5 & 4.1458 & 3.6276 \\
\hline
\end{tabular}

Table 5.1: Comparison between the boundaries of stability for the type II $\mathrm{D} p$-branes $\mathrm{n}$ the weak and strong coupling limits of $\lambda$ and for the M-branes in the $N=1$ and $N \rightarrow \infty$ limits.

limit, which is easily extended to the spinning case [1]1]. In particular, for the D-branes with $N$ fixed but with $\lambda$ varying between the two limits, the conjecture reads

$$
F_{\lambda}\left(T,\left\{\Omega_{i}\right\}\right)=f\left(\lambda, T,\left\{\Omega_{i}\right\}\right) F_{\lambda=0}\left(T,\left\{\Omega_{i}\right\}\right)
$$

where $F_{\lambda=0}\left(T,\left\{\Omega_{i}\right\}\right)$ is the free energy for $\lambda=0$. For M-branes the conjectures involves a function interpolating between $N=1$ and any finite $N$. An important first check of this conjecture is the fact that the free energies of the D-branes in the two limits show the same $N^{2}$ factor in front. This implies that only string loop corrections, which carry factors of $\frac{1}{N^{2}}$ would modify this behavior, and thus do not have to be considered in the large $N$ limit.

As a corroboration of this conjecture, it is seen from Table that the critical values of the dimensionless quantity $\Omega / T$ for the D2, D3, D4, M2 and M5-branes are remarkably close in the weak and strong coupling limit: It is hence plausible that in these cases the conjectured interpolation between the two limits holds. For the D1 and D6-brane case, however, there cannot be a smooth transition and a boundary of stability is somehow created/destroyed at some special point between the two limits. Finally, for the D5-brane we see that moving away from strong coupling at some point the phase space must expand, since at weak coupling $T$ and $\Omega_{i}$ are again independent thermodynamic quantities.

Turning to the second test, we recall that the 
string loop expansion in $g_{s}$ and derivative expansion in $\alpha^{\prime}=l_{s}^{2}$ translates through the AdS/CFT correspondence into a $1 / N$ and $1 / \lambda$ expansion respectively. The tree-level higher derivative term $l_{s}^{6} R^{4}$ of the type II string effective action can thus be used to compute the $\lambda^{-3 / 2}$ corrections to the free energy of the D-branes, which was analyzed for the non-rotating D3-brane in [1 15$]$. For simplicity we focus on the spinning D3-brane with one non-zero angular momentum, for which the weak and strong coupling free energies are respectively given by,

$$
\begin{aligned}
& F_{\lambda=0}(T, \Omega)=-N^{2} V_{3} T^{4}\left(\frac{\pi^{2}}{6}+\frac{1}{4} \omega^{2}-\frac{1}{32 \pi^{2}} \omega^{4}\right) \\
& F_{\lambda=\infty}(T, \Omega) \\
& =-N^{2} V_{3} T^{4}\left(\frac{\pi^{2}}{8}+\frac{1}{8} \omega^{2}+\frac{1}{16 \pi^{2}} \omega^{4}+\ldots\right)
\end{aligned}
$$

Since the dual theory, $\mathrm{N}=4 D=4 \mathrm{SYM}$, is con-

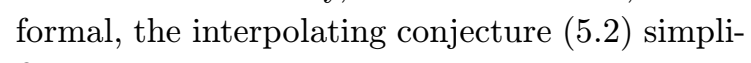
fies to

$$
F_{\lambda}(T, \Omega)=f(\lambda, \omega) F_{\lambda=0}(T, \Omega)
$$

From $(5.3) f(\lambda, \omega)$ is thus expected to be smaller than one, decreasing with $\lambda$, for fixed $\omega<1$, since $-F_{\lambda=\infty}<-F_{\lambda=0}$ for $\omega<1$. This has been corroborated in [1] $\left.1_{1}^{1}\right]$ by computing the $R^{4}$ corrections to the free energy from the spinning D3-brane solution (12.7).

\section{Spinning brane bound states and noncommutative field theories}

We finally comment on the generalization involving bound states of spinning branes, which are string backgrounds with a non-zero NSNS $B$-field (or $\mathcal{C}$-field in the case of M-theory). These have recently attracted much attention, in view of the appearance of non-commutative geometry in certain limits of such backgrounds, as first discovered in the context of $\mathrm{M}($ atrix) theory [1 $[1]$ specifically, non-commutative super Yang-Mills (NCSYM) appears [1] in a special low-energy limit of the world-volume theory of $N$ coinciding $\mathrm{D} p$-branes in the presence of a NSNS $B$-field. This fact has been used to extend the correspondence between near-horizon $\mathrm{D} p$-brane supergravity solutions and super Yang-Mills (SYM) theo-

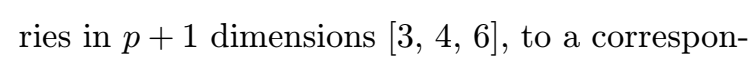
dence between near-horizon $\mathrm{D} p$-brane supergravity solutions with a non-zero NSNS $B$-field and

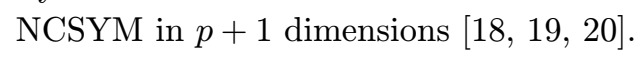

By applying a sequence of $\mathrm{T}$-dualities on the general spinning D-brane solution (2. $\overline{2} . \overline{1})$ one obtains the general spinning D-brane bound state solutions [12 2]. The resulting backgrounds are bound states of spinning $D(p-2 k)$-branes, $k=$ $0 \ldots m$, with $2 m \leq p$ the rank of the NSNS $B$ field,

$$
\begin{aligned}
B_{2 k-1,2 k} & =\tan \theta_{k}\left(H^{-1} D_{k}-1\right) \\
D_{k} & =\left(\sin ^{2} \theta_{k} H^{-1}+\cos ^{2} \theta_{k}\right)^{-1}
\end{aligned}
$$

where $k=1 \ldots m$ and we refer to [1]2] for the explicit form of the complete solution. The extra parameters that are introduced are the angles $\theta_{k}$, $k=1 \ldots m$. Besides the charges and chemical potentials of the D-branes in the bound states, which depend on these angles, the thermodynamic quantities of the bound state solution are not affected by the non-zero $B$-field and coincide with those of the spinning $\mathrm{D} p$-brane given in $(2.9)$.

The construction of the near-horizon limit of these solutions uses the rescaling $\left(\frac{2}{2} \cdot 6 i\right)$, and takes the angles $\theta_{k}$ to $\frac{\pi}{2}$ while keeping fixed

$$
b_{k}=l_{s}^{2} \tan \theta_{k}
$$

which are the non-commutativity parameters entering the position commutators on the worldvolume of the brane

$$
\left[y^{2 k-1}, y^{2 k}\right] \sim b_{k}, \quad k=1 \ldots m
$$

The dual field theories is then NCSYM in $p+1$ dimensions with effective coupling constant

$$
z \equiv g_{\mathrm{eff}}^{2}=g_{\mathrm{YM}}^{2} N\left(\prod_{k=1}^{m} b_{k}\right) r^{p-3}
$$

It turns out that the gauge coupling phase structure exhibits a far richer structure than the com-

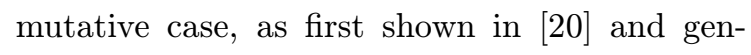
eralized in [12]']. To study this we set $b_{k}=b$, $k=1 \ldots m$ and consider a general path in phase space, parametrized by

$$
g_{\mathrm{YM}}^{2} \propto z^{\alpha}, \quad r \propto z^{\beta}, \quad b \propto z^{\gamma}
$$


keeping $N$ fixed, and where $(6.5)$ constrains the scaling exponents to satisfy $\alpha+(p-3) \beta+m \gamma=1$. The case $\alpha=\gamma=0$ in $(6.6)$ corresponds to the one described in [200]. It then follows that the supergravity description is valid provided [1 12 ?

$$
z \ll N^{\frac{4}{7-p}}\left(1+\left(\frac{z}{z_{\mathrm{nc}}}\right)^{\eta}\right)^{\frac{2 m}{7-p}}
$$

where we have used that

$$
b^{2}\left(\frac{r}{h}\right)^{7-p}=\left(\frac{z}{z_{\mathrm{nc}}}\right)^{\eta}, \eta=4 \beta+2 \gamma-1
$$

Depending on the values of $\eta$ and $z_{\text {nc }}$ this condition can then be shown to generate four types of phase diagrams, and for each spatial worldvolume dimension of the brane and each non-zero rank of $B$-field, a path and region of phase space can be chosen such that the phase structure of any of the four phase diagrams can be realized [1는. These exhibit various interesting features, including regions in which the supergravity description is valid for finite $N$ and/or the effective coupling is allowed to range from the transition point $g_{\text {eff }} \sim 1$ all the way to infinity.

As was argued for the non-rotating case [1]

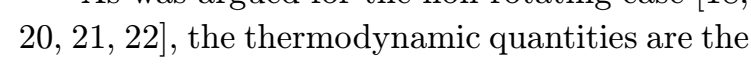
same as for commutative SYM case up to the replacement

$$
g_{\mathrm{YM}}^{2} \rightarrow g_{\mathrm{YM}}^{2} \prod_{k=1}^{m} b_{k}
$$

where $g_{\mathrm{YM}}$ on the left-hand side is the YM coupling constant of the commutative theory and $g_{\mathrm{YM}}$ on the right-hand side of the non-commutative theory ${ }^{\ddagger 2}$. This was argued at weak coupling from the field theory point of view [23] by showing that the planar limit of SYM and NCSYM coincide. As an application of the general phase structure analysis, the validity of the thermodynamics for the NCSYM has been examined by requiring that the intensive thermodynamic parameters are invariant for the path in phase space. This condition corresponds to the choice $\alpha+m \gamma=(5-p) \beta$ in (66.6i). In particular, this enables determining the region of phase space in

\footnotetext{
$\ddagger 2$ For the noncommutative D3-brane case, tree-level $R^{4}$ corrections to the thermodynamics have been addressed in [2] 2 2] $]$.
}

which $N$ can be finite and at the same time the coupling can be taken all the way to infinity. The resulting condition is that [1]

$$
T \gg b^{-1 / 2}\left(\frac{r_{0}}{r_{H}}\right)^{\frac{7-p}{2}}
$$

showing that at fixed non-extremality parameter $r_{0}$ and horizon radius $r_{H}$, the larger the noncommutativity parameter $b$, the larger the temperature region in which these properties are satisfied.

The presence of non-zero angular momenta does not qualitatively change the gauge coupling phase structure, but may well provide further insights into NCSYM in the presence of voltages for the R-charges. Moreover, in view of the recent discovery that the D6-brane theory with $B$ field decouples from gravity [11 $18,2 \overline{2}, \overline{2} 0]$ it is interesting that while the non-rotating case is thermodynamically unstable, for the spinning D6-brane stability is found in the canonical ensemble for sufficiently high angular momentum density We refer to [122] for a detailed treatment of the thermodynamics of this case.

We finally comment on some issues related to the possibility of finite $N$. In the non-commutative case finite $N$ is possible when the non-commutativity parameter $a^{\text {eff }}$ is large, so that the $1 / N$ expansion $^{\ddagger 3}$ turns into an expansion in $1 / a^{\text {eff }}$ in that case [1 127 . This suggests that for finite $N$ and large $a^{\text {eff }}$ planar diagrams dominate; indeed this has recently been shown to hold in perturbative field theory [205in]. The fact that finite $N$ is possible also fits together with the recent observation [2] that there are infinitely many $\mathrm{D}(p-2)$-branes in the near-horizon limit of the $\mathrm{D}(p-2)-\mathrm{D} p$ system, which means that the world volume theory of the $\mathrm{D}(p-2)$-brane has gauge group $U(\infty)$.

\section{Acknowledgments}

We thank the organizers of the TMR network meeting Quantum aspects of gauge theories, supersymmetry and unification in Paris for a very pleasant and interesting workshop.

\footnotetext{
${ }^{\ddagger}$ For a discussion of $1 / N$ corrections to the NCSYM thermodynamics, see [21] for an analysis at strong coupling and [2] for a field theory analysis.
} 


\section{References}

[1] J. D. Bekenstein, Black holes and entropy, Phys. Rev. D7 (1973) 2333-2346; S. W. Hawking, Particle creation by black holes, Commun. Math. Phys. 43 (1975) 199.

[2] G. T. Horowitz and A. Strominger, Black strings and p-branes, Nucl. Phys. B360 (1991) 197-209;

[3] J. Maldacena, The large $N$ limit of superconformal field theories and supergravity, Adv. Theor. Math. Phys. 2 (1998) 231-252, hep-th/9711200i].

[4] N. Itzhaki, J. M. Maldacena, J. Sonnenschein, and S. Yankielowicz, Supergravity and the large $N$ limit of theories with sixteen supercharges, Phys. Rev. D58 (1998) 046004, hep-th/9802042i].

[5] G. 't Hooft, A planar diagram theory for strong interactions, Nucl. Phys. B72 (1974) 461.

[6] O. Aharony, S. S. Gubser, J. Maldacena, H. Ooguri, and Y. Oz, Large $N$ field theories, string theory and gravity, 'hep-th/9905111;

[7] M. J. Duff, TASI lectures on branes, black holes and Anti-de Sitter space, hep-th/9912164.

[8] S. S. Gubser, Thermodynamics of spinning D3-branes, Nucl. Phys. B551 (1999) 667, hep-th/9810225].

[9] R.-G. Cai and K.-S. Soh, Critical behavior in the rotating D-branes, Mod. Phys. Lett. A14 (1999) 1895-1908, [hep-th/9812121].

[10] M. Cvetic and S. S. Gubser, Thermodynamic stability and phases of general spinning branes, JHEP 07 (1999) 010, "hep-th/9903132i.

[11] T. Harmark and N. A. Obers, Thermodynamics of spinning branes and their dual field theories, JHEP 01 (2000) 008, [hep-th/9910036i.

[12] T. Harmark and N. A. Obers, Phase structure of non-commutative field theories and spinning brane bound states, hep-th/9911169'.

[13] M. Cvetic et. al., Embedding AdS black holes in ten and eleven dimensions, Nucl. Phys. $\mathbf{B 5 5 8}$ (1999) 96-126, [hep-th/9903214i].

[14] R. C. Myers and M. J. Perry, Black holes in higher dimensional space-times, Ann. Phys. 172 (1986) 304. M. Cvetic and D. Youm, Near BPS saturated rotating electrically charged black holes as string states, Nucl. Phys. B477 (1996) 449-464, [hep-th/9605051];
[15] S. S. Gubser, I. R. Klebanov, and A. A. Tseytlin, Coupling constant dependence in the thermodynamics of $N=4$ supersymmetric Yang-Mills theory, Nucl. Phys. B534 (1998) 202, [hep-th/9805156].

[16] A. Connes, M. R. Douglas, and A. Schwarz, Noncommutative geometry and matrix theory: Compactification on tori, JHEP 02 (1998) 003, hep-th/9711162].

[17] N. Seiberg and E. Witten, String theory and noncommutative geometry, JHEP 09 (1999) 032, [hep-th/9908142].

[18] J. M. Maldacena and J. G. Russo, Large $N$ limit of non-commutative gauge theories, JHEP 09 (1999) 025, hhep-th/9908134i .

[19] A. Hashimoto and N. Itzhaki, Non-commutative Yang-Mills and the AdS/CFT correspondence, hep-th/9907166; M. Li and Y.-S. Wu, Holography and noncommutative Yang-Mills, ihep-th/9909085!.

[20] M. Alishahiha, Y. Oz, and M. M. Sheikh-Jabbari, Supergravity and large $N$ noncommutative field theories, hep-th/9909215.

[21] J. L. F. Barbon and E. Rabinovici, On $1 / N$ corrections to the entropy of noncommutative Yang-Mills theories, hep-th/9910019;

[22] R.-G. Cai and N. Ohta, On the thermodynamics of large $N$ noncommutative super Yang-Mills theory, hep-th/9910092.

[23] D. Bigatti and L. Susskind, Magnetic fields, branes and noncommutative geometry, hep-th/9908056.

[24] G. Arcioni and M. A. Vazquez-Mozo, Thermal effects in perturbative noncommutative gauge theories, JHEP 01 (2000) 028, hep-th/9912140i].

[25] S. Minwalla, M. V. Raamsdonk, and N. Seiberg, Noncommutative perturbative dynamics, hep-th/9912072.

[26] J. X. Lu and S. Roy, $(p+1)$-dimensional noncommutative Yang-Mills and $D(p-2)$ branes, ihep-th/9912165i R.-G. Cai and N. Ohta, Noncommutative and ordinary super Yang-Mills on $(D(p-2), D p)$ bound states, hep-th/0001213. 